\title{
Modelling, Speckle Simulation and Quality Evaluation of Synthetic Ultrasound Images
}

\author{
Prerna Singh $^{1 *}(0000-0003-2770-9493)$, Ramakrishnan Mukundan ${ }^{1}(0000-0003-4578$ - \\ 1931), Rex de Ryke²(0000-0002-2577-899X) \\ ${ }^{1}$ Department of Computer Science and Software Engineering \\ University of Canterbury, Christchurch, New Zealand. \\ prerna.singhepg.canterbury.ac.nz \\ mukundandcanterbury.ac.nz \\ ${ }^{2}$ Radiology Services, Christchurch District Health Board \\ Christchurch, New Zealand. \\ Rex.DeRyke@cdhb.health.nz
}

\begin{abstract}
Speckle noise reduction is an important area of research in the field of ultrasound image processing. Several algorithms for speckle noise characterization and analysis have been recently proposed in the area. Synthetic ultrasound images can play a key role in noise evaluation methods as they can be used to generate a variety of speckle noise models under different interpolation and sampling schemes, and can also provide valuable ground truth data for estimating the accuracy of the chosen methods. However, not much work has been done in the area of modelling synthetic ultrasound images, and in simulating speckle noise generation to get images that are as close as possible to real ultrasound images. This paper discusses these aspects, presents novel algorithms for speckle simulation and modelling based on three sampling schemes, and also evaluates the quality of the outputs using image quality metrics. Detailed experimental analysis including both quantitative and subjective assessments are also presented.
\end{abstract}

Keywords: Ultrasound image analysis. Speckle simulation. Speckle noise reduction. Synthetic ultrasound images. Image quality assessment.

\section{Introduction}

Ultrasound images are known to have poor signal-to-noise ratio, yet they are low cost, non-invasive techniques in diagnostic radiology and hence extensively used in clinical applications. Several new ultrasound image analysis algorithms are currently being researched for noise reduction [1-3], segmentation [4], registration and volume reconstruction [5]. Online ultrasound image databases are now becoming increasingly available and this has greatly benefitted researchers in obtaining reference images for testing and evaluating algorithms [5-7].

adfa, p. 1, 2011.

(C) Springer-Verlag Berlin Heidelberg 2011 
The speckle noise in ultrasound images degrades the fine details and edge definitions, and limits the contrast resolution by making it difficult to detect small and low contrast lesions in the body. Therefore, algorithms for ultrasound image filtering and analysis primarily focus on the characteristics of speckle noise and try to minimize its effects on image interpretation [8]. To analyse the effectiveness or accuracy of speckle reduction techniques, it is necessary to add controlled noise to ideal noiseless images [2]. In the absence of such noiseless ground truth images, researchers commonly use standard non-ultrasound test images (eg. Lena, Mandrill etc.), model speckle noise on those images and perform algorithm evaluation. This paper addresses the need for generating accurate synthetic models of ultrasound image formation for applications in speckle noise analysis. A synthetic ultrasound image can be sampled using a configuration of points that correspond to either linear or sector scan modes of ultrasound imaging, and interpolated later after generating speckle noise at the sampled points to obtain visually realistic effects. Synthetic images can therefore be used to generate simulated ultrasound images with a wide range of image and noise characteristics useful for filtering methods and noise analysis.

Statistical and empirical methods of generating speckle lack realism owing to the lack of image modeling. There are only very limited algorithms reported in literature for speckle simulation based on image acquisition modeling. Perreault and AuclairFortier [9] proposed an efficient simulation model of ultrasound images based on a radial-polar configuration of sampling points and a speckle noise simulation algorithm. We extend their work by considering different types of sampling and interpolation schemes and by performing detailed experimental analysis to compare their effectiveness in producing realistic speckle simulation. Their work used images of Lena and Barbara for generating the simulated images with speckle noise. However, for generating highly realistic synthetic models, we require images that clearly show the anatomical features present in an ultrasound image without the noise content. To achieve this goal, we used an artist to render the features based on three reference ultrasound images, and used this as our base image.

A very important aspect of synthetic image modelling algorithms is quality assessment. In the proposed method, the base synthetic image is modified as outlined above using the acquisition model, speckle noise simulation and interpolation of the sampled points. To the authors knowledge, no prior work has been reported on image quality assessment of ultrasound images using spatial frequency measure (SFM), and spectral activity measure (SAM) metrics. In this paper, the quality of the generated outputs is compared with that of real ultrasound images using these image quality metrics.

The paper presents the complete framework for the development of synthetic ultrasound images including the set of processes in both simulation and evaluation stages. Each stage incorporates a wide range of parametric variations and options, allowing the user to generate synthetic images with varying levels of sampling, interpolation and noise characteristics. This paper is organized as follows: The next section gives a brief outline of the images used and the methods in the processing pipeline. Section 3 describes the simulation model in detail. Section 4 presents the methods used in the evaluation stage. Section 5 presents experimental results and their evaluations, and 
Section 6 gives a summary of the work presented in the paper and outlines future directions.

\section{Materials and Methods}

All experimental work presented in the paper are based on images derived from three reference ultrasound images sourced from the online ultrasound image gallery [7]. These are ultrasound scans of the liver, and have very similar image features, intensity distribution and noise content. Three reference images were used because ultrasound images could present variations in texture, image quality and speckle noise content as shown by the SFM and SAM values in Table 1 later in Section 5. For comparison, the images and their histograms are presented in Fig. 1.
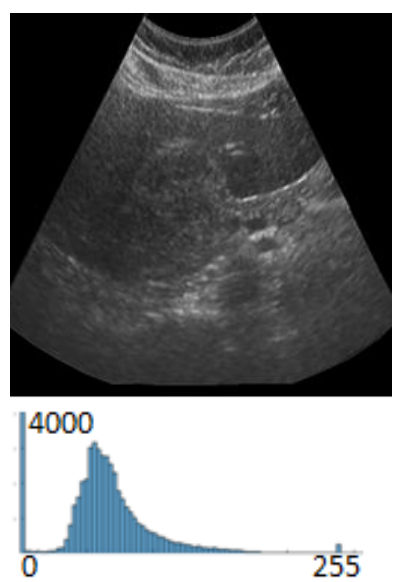

Liver-1
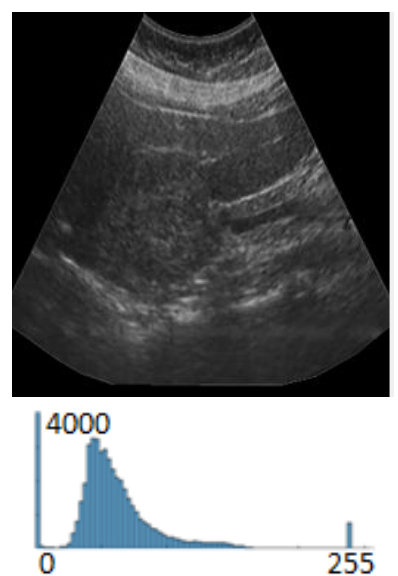

Liver-2
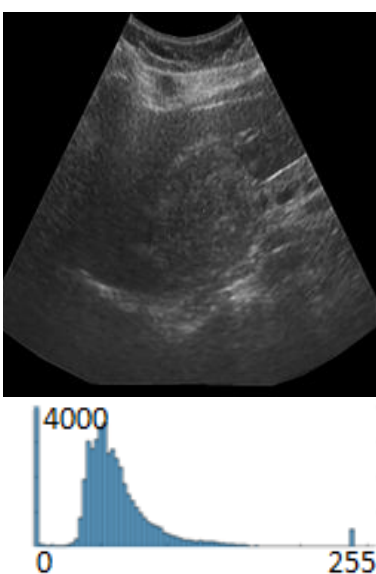

Liver-3

Fig. 1. Reference ultrasound images [7] used in our work, and their histograms.

The three reference images in Fig. 1 were used by an artist to sketch the image features which formed our base synthetic image (Fig. 2). The histogram of the synthetic image bears similarity with those of the reference images.
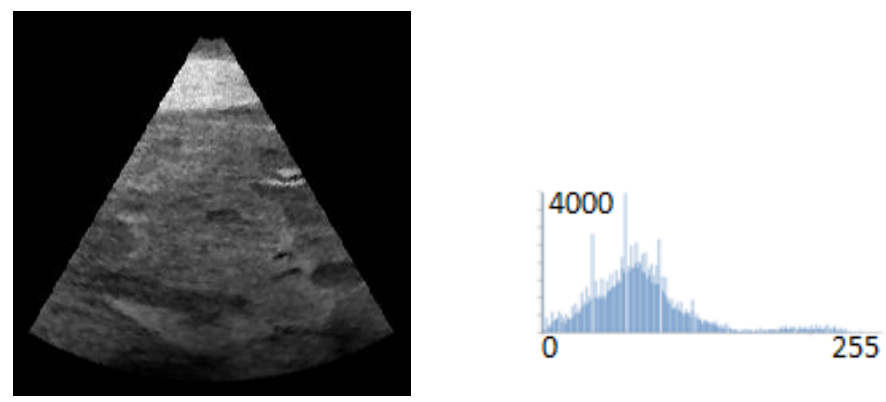

Fig. 2. Artist rendered synthetic image and its histogram. 
The main methods used in the speckle simulation modelling and evaluation pipeline are depicted in Fig. 3. Within the simulation model, the synthetic image is first sampled based on an acquisition model, speckle noise is then generated at the sampled points, and an interpolation algorithm used to fill the sector scan region. The evaluation model uses image quality metrics computed for the output are then compared with those of the reference ultrasound images for a quantitative assessment of the quality of the final synthetic images. A subjective evaluation is also performed using expert sonographers.

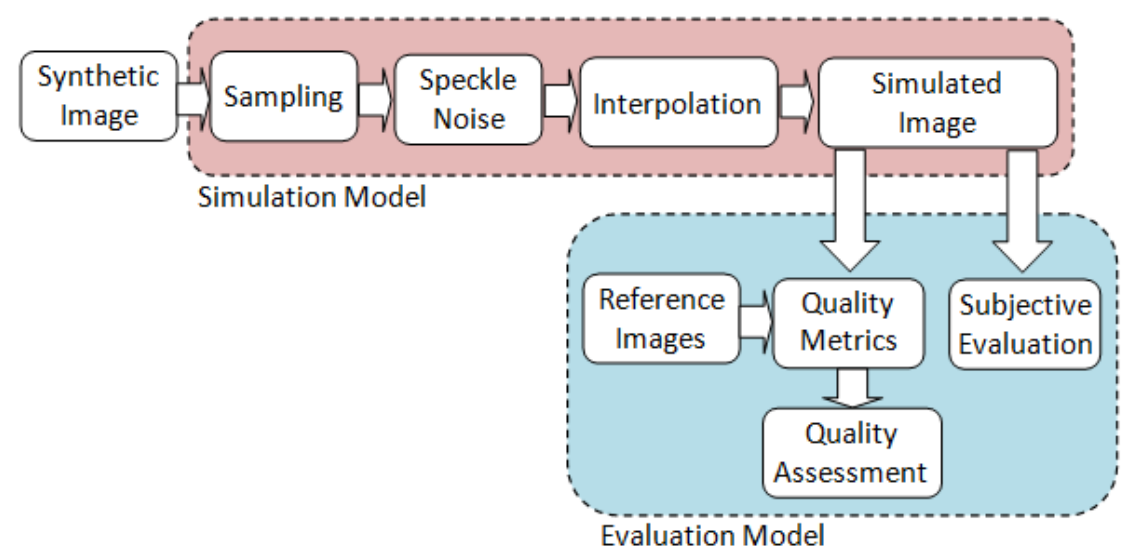

Fig. 3. The simulation and evaluation stages of the processing pipeline.

The processing stages within the simulation and evaluation models are further elaborated in the following sections.

\section{The Simulation Model}

The first stage of the sampling model is the method that generates a set of points at a coarse spatial resolution. The configuration of points models the loss of resolution of the ultrasound image due to pulse length, and also the scanning mode (sector or linear). One of the original contributions in this field is the paper by Perreault and Auclair-Fortier [9], where a radial-polar sampling model was introduced. We extend their work and propose three types of sampling methods called radial-polar, radialuniform, and uniform grid. The first two are closely related to sector scan, while the third corresponds to a sampling in linear orthogonal directions (Fig. 4). 


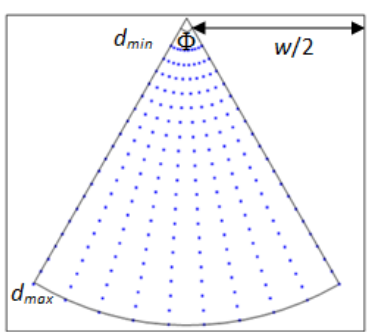

Radial-Polar

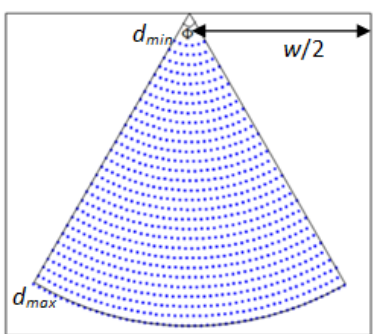

Radial-Uniform

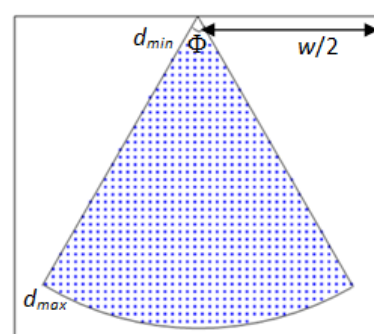

Uniform Grid

Fig. 4. Sampling models that can be used in simulating speckle noise.

In Fig. 4, the sector angle is denoted by $\Phi$, and the extent of the sector is given by radial distances $d_{\min }$ and $d_{\max }$. The image width is denoted by $w$. We also denote the total number of divisions along each radial line (axial resolution) by $m$, and the number of division of the sector angle (lateral resolution) by $n$. The Cartesian coordinates of the sampled points for radial-polar sampling are given by

$$
\begin{gathered}
d_{j}=d_{\min }+j\left(d_{\max }-d_{\min }\right) /(m-1) ; \quad \theta_{i}=(3 \pi-\Phi) / 2+i \Phi /(n-1) \\
x(i, j)=d_{j} \cos \theta_{i}+w / 2 ; \quad y(i, j)=-d_{j} \sin \theta_{i} ; \\
i=0 . .(n-1) ; \quad j=0 . .(m-1)
\end{gathered}
$$

The non-uniform spacing of points in the radial-polar sampling method causes the density of points to increase towards the sector's apex. The radial-uniform sampling method uses a constant arc length $\Delta$ between points along each arc to generate a uniform spacing between points. The equations for this sampling model are same as in eq. (1) except that the polar angle $\theta$ will now depend on both $i$ and $j$ as shown below.

$$
\theta_{i j}=(3 \pi-\Phi) / 2+i \Delta / d_{j}
$$

The uniform grid is the simplest sampling model corresponding to a rectangular arrangement of uniformly spaced points with a constant distance $\delta$ between points. If a sector scan region is required, the points outside the region are clipped using the line equations of the two bounding edges. Using eq.(3), if $f\left(x, y, \theta_{\min }\right)>0$ or $f\left(x, y, \theta_{\max }\right)$ $<0$, the point $(x, y)$ is outside the sector region.

$$
\begin{gathered}
\theta_{\text {min }}=(3 \pi-\Phi) / 2 ; \quad \theta_{\max }=(3 \pi+\Phi) / 2 \\
f(x, y, \theta)=(x-w / 2) \sin \theta+y \cos \theta
\end{gathered}
$$

More details and implementation aspects of the above three models are given in [10]. For speckle simulation, we use the method given in [7]. Their model is based on a complex distribution of incoherent phasors $(u, v)$ given by a two-dimensional Gaussian function $g_{\sigma}$. The complex amplitude of each pixel is initialized with the square-root of the sampled intensity value. The number of incoherent phasors $M(x, \mathrm{y})$ at each pixel $(x, y)$ is set as the value of a random number under a uniform distribution 
within a pre-specified range $[a, b]$. The incoherent phasors are generated and added $M$ times to both the real and imaginary components of the complex value at each pixel. The noisy intensity value is then given by the amplitude of the complex number.

After generating speckle noise at the sampled points, we use an interpolation method to fill the empty space left by the sampling step. In general, the interpolated value at a specified coordinate $(x, y)$ of an image $I$ is computed by grouping the sample values at neighboring pixels $(l, m)$ using the following formula [11]:

$$
I(x, y)=\sum_{l, m \in Z} \varphi(x-l, y-m) I(l, m)
$$

where, $\varphi()$ denotes a two-dimensional interpolation/synthesis function that provides the weights of the linear combination of sampled intensity values. Commonly used interpolation methods are B-Spline and cubic Hermite [11, 12]. In [9], the authors used an interpolation scheme using the Lanczos-3 kernel [13, 17].

\section{The Evaluation Model}

One of the key requirements in the analysis of image modelling and simulation algorithms that use synthetic data is image quality assessment. Image quality metrics are also extensively used in the evaluation of compression and noise filtering algorithms [14]. In this paper, we use the following three quality measures: $E, S F M$ and SAM.

Entropy: It measures the degree of randomness in an image, and is defined as

$$
E=-\sum_{j} P_{j} \log _{2} P_{j}
$$

where, $P_{j}$ is the probability associated with gray level $j$, and is usually computed as the ratio of the histogram value of the intensity $j$ to the total number of pixels.

Spatial Frequency Measurement (SFM) is a way to measure the overall activity level in an image. SFM is expressed as,

$$
S F M=\sqrt{g_{R}+g_{C}}
$$

where, $g_{R}$ and $g_{C}$ denote the mean pixel-level intensity gradients along rows and columns evaluated on an image of size $M \times 1 N$ pixels as given below [16]:

$$
\begin{aligned}
& g_{R}=\sqrt{\frac{1}{M N} \sum_{i=0}^{M-1} \sum_{j=1}^{N-1}(I(i, j)-I(i, j-1))^{2}} \\
& g_{C}=\sqrt{\frac{1}{M N} \sum_{i=1}^{M-1} \sum_{j=0}^{N-1}(I(i, j)-I(i-1, j))^{2}}
\end{aligned}
$$


The spectral activity measure (SAM) is a measure of image predictability (higher values indicating higher predictability). For an image of size $M \mathrm{x} N$ pixels, it is defined in terms of the Discrete Fourier Transform (DFT) coefficients of the image in the frequency domain as follows:

$$
S A M=\frac{\frac{1}{M \cdot N} \sum_{j=0}^{M-1} \sum_{k=0}^{N-1}|F(j, k)|^{2}}{\left[\prod_{j=0}^{M-1} \prod_{k=0}^{N-1}|F(j, k)|^{2}\right]^{\frac{1}{M \cdot N}}}
$$

where, $F(j, k)$ denotes the DFT coefficient at position $(j, k)[16]$.

Other traditionally used objective measures such as mean square error (MSE), peak signal-to-noise ratio (PSNR) and speckle index (SI) [15] are useful only in the context of noise reduction and filtering algorithms. For example, the SI value measures the level of residual speckle noise in an image, and is therefore not a useful measure in a synthetic image modelling application.

The values of the entropy, SFM, SAM computed for the three reference images (Liver-1, Liver-2, Liver-3 given in Fig. 1) are shown below in Fig. 5. From the SFM and SAM values, it can be seen that the reference images have higher predictability and less details, as is common in ultrasound images.

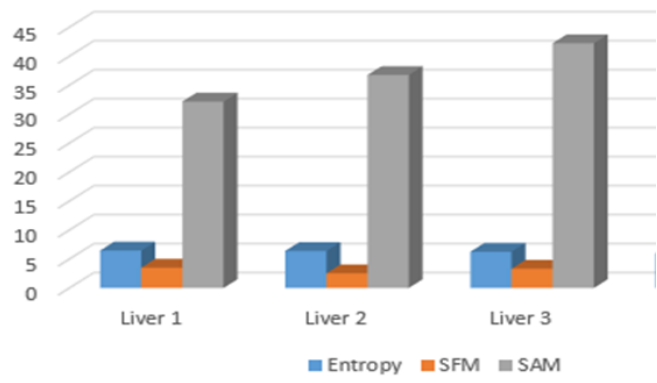

Fig. 5. Entropy, SFM, SAM values of the reference images.

In addition to using the above objective measures, we also propose to use subjective assessment of quality by clinical experts in our evaluation model. The importance of subjective evaluation in image quality assessments is emphasized in [16]. The outputs of the speckle simulation stage were assessed by clinical experts, and their subjective evaluations are discussed in the next section.

\section{$5 \quad$ Experimental Results and Analysis}

The proposed framework provides allows several options and parametric variations in each stage of the pipeline. As seen in Section 3, the three sampling methods and 
three interpolation schemes themselves give nine possible combinations. Each sampling scheme has its own set of parameters that can be varied over a wide range of values. The speckle noise generation algorithm also has a set of statistical parameters governing the noise distribution. Due to limitation of space, only a few sample outputs are presented in this section. Further, subtle variations in image or noise characteristics cannot be clearly perceived when images are reduced to fit within a small space.

The first row of Fig. 6 shows the variations when the axial resolution $m$ is increased in radial-polar sampling, keeping the lateral resolution fixed at $n=40$. The interpolation used was Lanczos-3 [17].

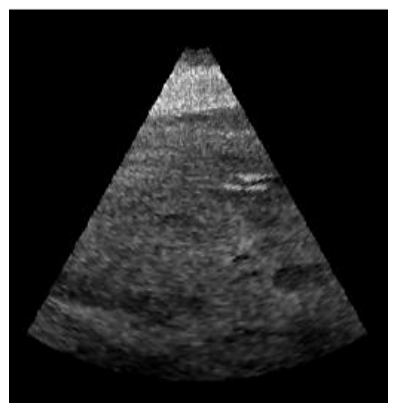

$m=120$

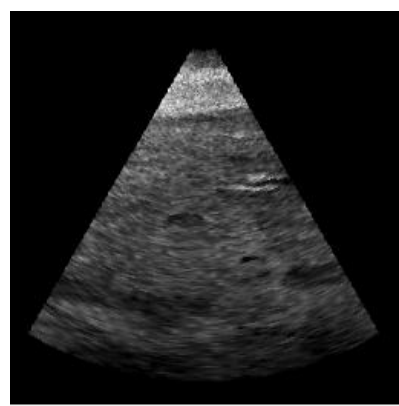

$m=160$

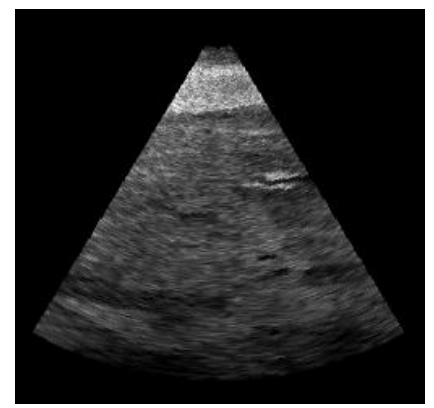

$m=200$

Fig. 6. Effect of changing axial resolution in radial-polar sampling.

Similar results for radial uniform sampling are shown in Fig. 7.

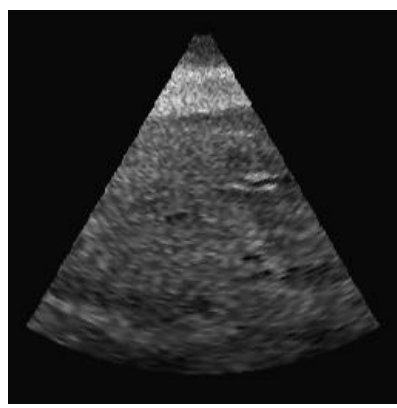

$m=120$

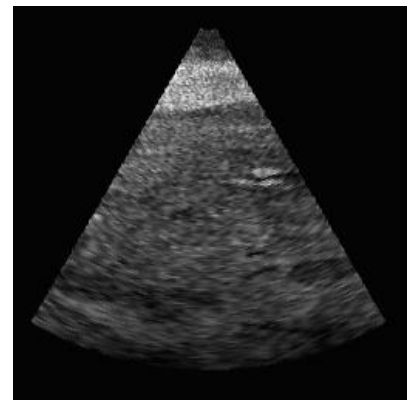

$m=160$

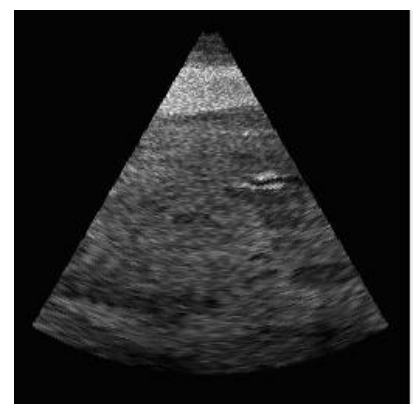

$m=200$

Fig. 7. Effect of changing axial resolution in radial-uniform sampling.

Some of the commonly found artifacts in simulated images when values of certain parameters become large are shown in Fig. 8. In Fig. 8(a), a large value for $m$ results in a dense, overlapping set of points along beam directions resulting in smoothing/merging of pixels. A similar effect is seen when both $n$ and $m$ are large (Fig. 8(b)). When the $\sigma$ value is large in the speckle generation function, the image becomes too grainy with loss of fine details, as in Fig. 8(c). 


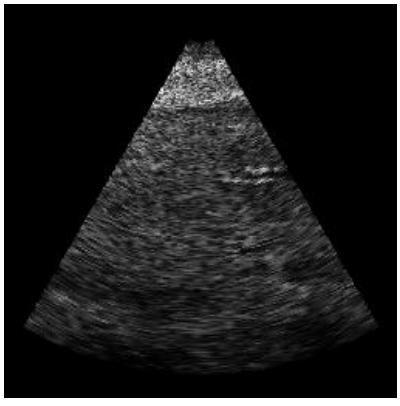

(a) $n=40, m=240, \sigma=1.7$

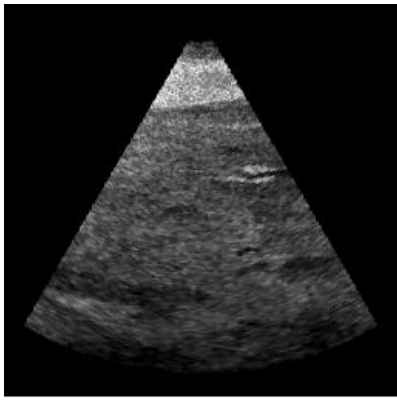

(b) $n=60, m=160, \sigma=0.5$

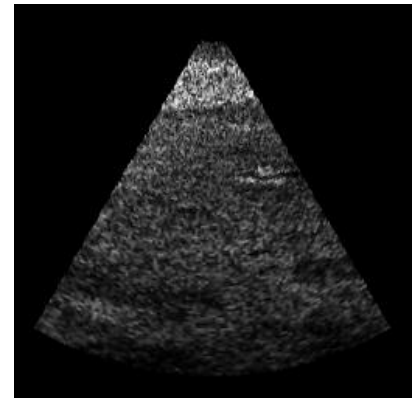

(c) $n=80, m=120, \sigma=1.9$

Fig. 8. Image artifacts produced by large values of sampling and noise parameters.

Fig. 9 gives a comparison of entropy, SFM and SAM values computed for the reference images and also the simulated images generated using various combinations of sampling and interpolation methods. The SAM values have been scaled by a factor of 10 to get a nearly uniform range of values for all three metrics. An important aspect to be considered while computing quality metrics is that the background pixels outside the sector region must be excluded from the computation.

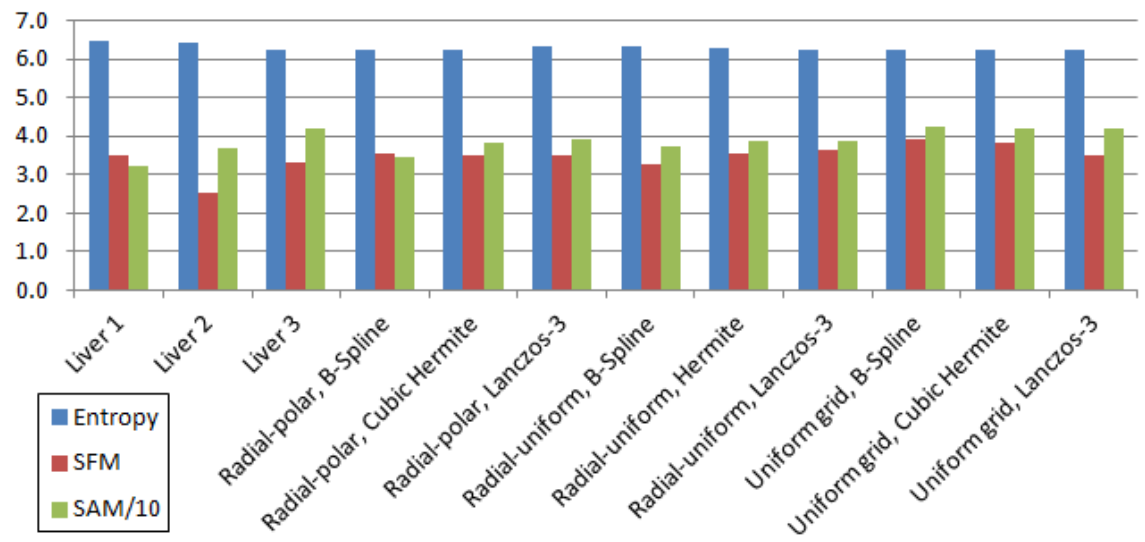

Fig. 9. A comparison of entropy, SFM and SAM values computed for the reference and synthetic images (SAM values scaled by a factor of 10).

Fig. 9 shows that the SFM and SAM values of the generated synthetic images are similar to that of the reference images on an average sense, which points to the fact that the synthetic image is visually similar to a real ultrasound image. The radialpolar sampling scheme with Lanczos-3 interpolation gave SFM values that are closest to the reference value. The SAM values showed larger range of variations. However, the values of all synthetic images were between the minimum and maximum of reference values. The uniform sampling method provided SAM values that are closest to 
that of the reference image. Four simulated synthetic ultrasound images which gave values closest to the reference values were shown to clinical experts for subjective evaluation. The images and their parameters are shown in Fig. 10.

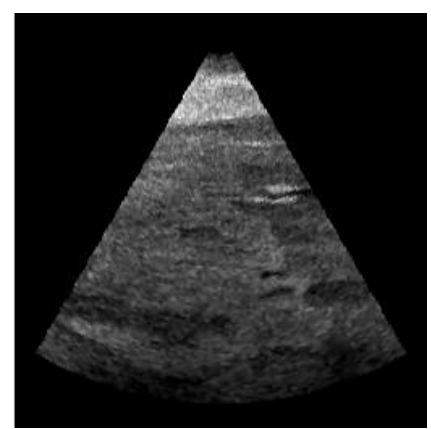

(a) $n=80, m=120, \sigma=0.5$

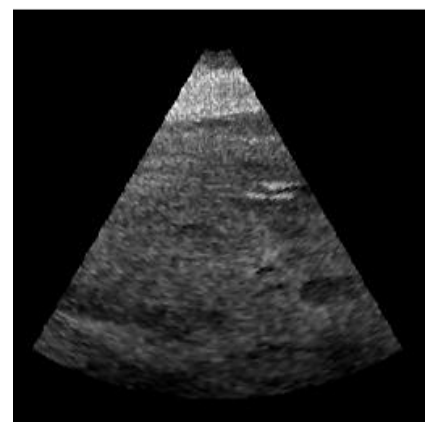

(c) $n=40, m=120, \sigma=0.5$

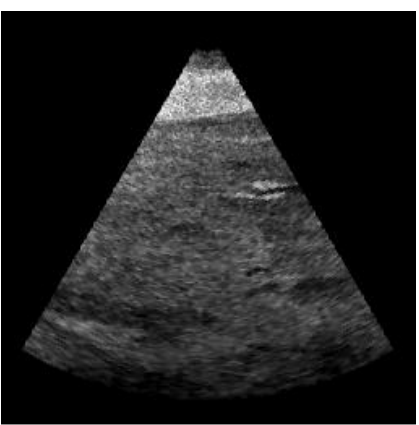

(b) $n=60, m=120, \sigma=0.5$

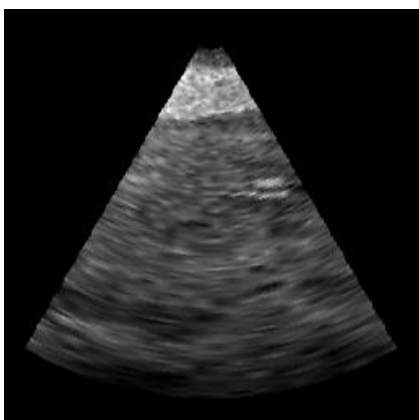

(d) $n=20, m=120, \sigma=0.5$

Fig. 10. Synthetic images with speckle noise used for subjective evaluation.

The subjective evaluation was performed by three experts. They based their evaluation on key visual features such as contrast, grayscale variations, texture and graininess. The images were scored on a scale from 1 to 5 . There was a general agreement among the assessors on image quality and how closely the simulated images resembled real ultrasound images. The mean subjective scores are given in Table 1 .

Table 1. Mean subjective evaluation scores assigned by clinical experts.

\begin{tabular}{l|c|c|c|c}
\hline Image & Fig. 10(a) & Fig. 10(b) & Fig. 10(c) & Fig. 10(d) \\
\hline Mean Score & 4 & 3 & 3 & 1 \\
\hline
\end{tabular}

As the lateral resolution parameter $n$ is varied from 20 to 80 , the smoothing effect due to interpolation is significantly reduced, the graininess improved and image features became more clearly visible, which is important from a clinician's perspective. The assessors also observed that the images have gray-scale variation and graininess closely resembling real ultrasound images. 
Overall, based on both quantitative and subjective evaluations, radial-polar sampling method with parameter values $n=80, m=120$ and speckle simulation with $\sigma=$ 0.5 with linear and Lanczos-3 interpolation gave the most accurate simulation of real ultrasound images, where the image size used was $256 \times 256$ pixels. In general, for an image of size $N \mathrm{x} N$ pixels, the optimal value $m$ of axial resolution (the number of pixels per beam) depends on the value of $N$. Our experiments by varying $N$ have shown that the optimal value of $m$ varies proportional to $N$ as follows:

$$
m=\text { floor }(0.56 N-23), \quad 250 \leq N \leq 500 .
$$

The value of the lateral resolution $n$ represents the number of ultrasound beams and it specifies the subdivisions of the sector angle. The optimal subdivision was found to be approximately 1 beam/degree, i.e., if the sector angle is $60 \mathrm{degs}$, the optimal value of $n$ is also around 60.

\section{Conclusions and Future Work}

This paper has presented the complete algorithmic framework for generating realistic and simulated ultrasound images incorporating image acquisition models, speckle noise formation processes and image interpolation schemes. These processes within the simulation model allows users to vary a wide range of parameters that control the image and noise formation processes. The simulated images with speckle noise could be used to evaluate noise filtering methods as ground truth data (the corresponding synthetic images without noise) are readily available.

The paper has introduced three sampling schemes, viz., radial-polar, radialuniform and uniform grid sampling methods. These methods together with the speckle simulation model and the interpolation scheme formed the simulation model of the processing pipeline. In the evaluation model, objective assessment of image quality was performed using entropy, SFM and SAM metrics. A subjective evaluation by clinical experts was also performed.

Experimental analysis shows that the synthetic image with simulated speckle noise has visual characteristics and image features very similar to real ultrasound images. The evaluation study helped to pick the best set of parameters that accurately modelled real ultrasound images, from a very large set of values.

Future work is directed towards more accurate content-specific modelling of speckle noise considering the regions present in the image, which will require additional processing such as region identification or segmentation.

\section{$7 \quad$ Acknowledgments}

We acknowledge the help extended by Dr. Khadijah Hajee Abdoula, Victoria Hospital, Quatre Bornes, Mauritius and Dr. Vivek Aggarwal, Thyroid Clinic, New Delhi, India, in providing their expert advice, valuable inputs and subjective evaluation of synthetic images produced in this research. 


\section{$8 \quad$ References}

1. Zhang J, Cui W, Wu L, Lin G, Cheng Y (2016) A novel algorithm based on wavelettrilateral filter for de-noising medical ultrasound images. In: Control and Decision Conference. doi: 10.1109/CCDC.2016.7531648

2. Malutan R, Terebes R, Germain C, Borda M, Cislariu M (2015) Speckle noise removal in ultrasound images using sparse code shrinkage. In: IEEE international conference on Ehealth and bioengineering. doi: 10.1109/EHB.2015.7391394

3. Le T (2014) Adaptive noise reduction in ultrasound imaging. In: IEEE symposium on signal processing in medicine and biology. doi: 10.1109/SPMB.2014.7002961

4. Zang X, Bascom R, Gilbert C, Toth J, Higgins W (2016) Methods for 2D and 3D endobronchial ultrasound image segmentation. IEEE Trans. on Biomedical Engineering 63(7) pp 1426-1439 doi: 10.1109/TBME.2015.2494838

5. Cortes C, Kabongo K, Macia I, Ruiz OE, Florez J (2015) Ultrasound image dataset for image analysis algorithms evaluation. In: Chen YW, Torro C, Tanaka S, Howlett R, Jain L. (eds) Innovation in Medicine and Healthcare 2015. Smart Innovation, Systems and Technologies, vol 45. Springer, Cham pp 447-457 doi: 10.1007/978-3-319-23024-5_41

6. Telmed Ultrasound Medical Systems. http://www.pcultrasound.com/products/products_usimg/index.html.

7. Ultrasound Image Gallery. http://www.ultrasound-images.com/.

8. Loizou CP, Pattichis CS (2015) Despeckle Filtering for Ultrasound Imaging and Video, Vol 1: Algorithms and Software. Morgan \& Claypool.

9. Perreault C, Auclair-Fortier MF. (2007) Speckle simulation based on B-mode echographic image acquisition model. In: 4th Canadian conference on computer and robot vision, pp 379-386 doi: 10.1109/CRV.2007.61

10. Singh P, Mukundan R, de Ryke R (2017) Synthetic models of ultrasound image formation for speckle noise simulation and analysis. In: International conference on signals and systems (ICSigSys-2017).

11. Kai-yu L, Wen-dong W, Kai-wen Z, Wen-bo L, Gui-li X (2014) The application of Bspline based interpolation in real-time image enlarging processing. In: 2nd International conference on systems and informatics pp 823-827.doi: 10.1109/ICSAI.2014.7009398

12. Goceri $E$, Lomenie $N$ (2010) Interpolation approaches and spline based resampling for MR images. In: 5th International symposium on health informatics and bioinformatics. doi: 10.1109/HIBIT.2010.5478891

13. Somawirata IK, Uchimura K, Koutaki G (2012) Image enlargement using adaptive manipulation interpolation kernel based on local image data. In: IEEE International conference on signal processing, communication and computing pp 474-478. doi: 10.1109/ICSPCC.2012.6335692

14. Xia ZW, Li Q, Wang Q (2012) Quality metrics of simulated intensity images of coherent ladar. In: International conference on optoelectronics and microelectronics. doi: 10.1109/ICoOM.2012.6316255

15. Mirza S, Kumar R, Shakher C (2005) Study of various preprocessing schemes and wavelet filters for speckle noise reduction in digital speckle pattern interferometric fringes. Optical Engineering 44(4). doi: 10.1117/1.1886749

16. Grgic S, Grgic M, Mrak M (2004) Reliability of objective picture quality measures. Jnl. of Elec. Engg. 55(1-2) pp 3-10. doi: 10.1.1.138.6936

17. Burger W, Burge MJ (2008) Digital Image Processing: An Algorithmic Introduction Using Java. Springer. doi: 10.1007/978-1-84628-968-2 